\title{
The Self-Healing Peculiarity of Airy Beams Propagation in Free-Space
}

\author{
Haitao Wang ${ }^{\mathrm{a}, \mathrm{b}}, *$, Chengyu Fan $^{\mathrm{a}}$ \\ ${ }^{a}$ Key Laboratory of Atmospheric Composition and Optical Radiation, Anhui Institute of Optics and Fine \\ Mechanics, Chinese Academy of Sciences, Hefei Anhui, 230031, China \\ ${ }^{b}$ State Key Laboratory of Pulsed Power Laser Technology, Electronic Engineering Institute, Hefei 230037,
}

China

\begin{abstract}
We have theoretically and numerically investigated the self-healing peculiarity of Airy beams truncated by different decay factors. The self-healing dynamic processes of truncated Airy beams are different from that of blocked Airy beams. The wing with large truncation of Airy beam can propagate much longer distance than that of small truncated wing. When one of the wings is complete truncated, the Airy beam propagates along a straight line and neither self-bending nor self-reconstruction can be observed. The Poynting vector and energy flow are conducted to gain an insight into the physical mechanism.
\end{abstract}

Keywords: (350.5500) Propagation; (050.1940) Diffraction; (260.1960) Diffraction theory; (260.2110) Electronmagnetic optics.

\section{Introduction}

Airy beams family as newly different class of non-diffracting beams, such as Bessel, Mathieu, Weber and discrete non-diffracting beams, have attracted much attention on their propagation and generation [1-4]. Besides the non-spreading feature, the remarkable peculiarities of Airy beams are that they do not propagate in a straight line path but along parabolic ballistic trajectory (self-bending), transversal acceleration, self-healing or self-reconstruction. Those properties offering a wide range of potential applications in the fields including plasma wave-guiding [5], microparticle manipulation [6], generation of light bullets and curved femto-filaments [7-8], and Generation of electron Airy beams [9].

Airy wave packet as the solution of the paraxial Schrödinger equation [10] was firstly observed by Berry and Balazs under the physical background of quantum mechanism [11]. Theoretically, the ideal Airy beam contains infinite energy. Although Airy function is not square integrabel [12], the gravity center remain invariant and the main lobe tends to accelerate along parabolic trajectory during propagation in spite of without external potential. The Airy beam can be seen as an interference pattern, similar to the Bessel beam [13-14]. Experimentally, the first optical realization of Airy beam by Siviloglou et al [15-16] is a truncation version, which carries finite energy and is generated from multiplying the Airy function distribution by an exponential decay factor or a Gaussian window. Recently, some other methods, such as using nonlinear processes in photonic crystal [17], or by utilizing constructed microchirp laser [18], 3/2 phase pattern [19] and so on, have been proposed for producing Airy beams.

Due to the intriguing propagation peculiarity of Airy beam (self-curving trajectory, weak diffraction and self-healing), many works have investigated and demonstrated the propagation dynamics of Airy beams from paraxial [20] to nonparaxial approximation [21-22] and from spatio-temporal 1D-2D to 3D-4D [23-24]. Many others Airy-type beams or pulses and beam array [25-27] have also triggered much attention, from linear media to the nonlinear regime [28-29]. Among those works, the self-accelerating and self-healing evolutions of Airy beams [30-31], whose main lobes are embed with a vortex or masked by an artificial presupposition particle, are interesting topics and have been widely investigated. While, if the two wings or one of them is truncated, whether or not the distinguishing processes of energy compensation and flow can occur to fulfill the self-accelerating and self-healing. This question has not been reported yet and remains an open question, but it will be investigated in the following arrangement.

\section{The theoretical description and mathematical equations}

We will start our analysis and get the ballistic dynamics of 2D Airy beams from the following basic paraxial Schrödinger equation [15, 30]:

$i \frac{\partial u(x, y, z)}{\partial z}+\frac{1}{2 k}\left(\frac{\partial^{2} u(x, y, z)}{\partial x^{2}}+\frac{\partial^{2} u(x, y, z)}{\partial y^{2}}\right)=0$,

where $k=2 \pi n_{0} / \lambda_{0}$ is the wavenumber and $\lambda_{0}=532 \mathrm{~nm}$ is the wavelength. The initial electric field envelope is given by: 


$$
\begin{array}{r}
u(x, y, z=0)=\prod_{m=x, y} \operatorname{Ai}\left(s_{m}\right) \exp \left(\mathrm{a}_{m} \mathrm{~s}_{m}\right) \\
=A i\left(\frac{x}{x_{0}}\right) \exp \left(\mathrm{a}_{x} \frac{x}{x_{0}}\right) A i\left(\frac{y}{y_{0}}\right) \exp \left(\mathrm{a}_{y} \frac{y}{y_{0}}\right) .
\end{array}
$$

The evolution pattern of accelerating Airy beam in closed form can be obtained from:

$$
\begin{gathered}
u(x, y, z)=\prod_{m=x, y} u_{m}\left(s_{m}, \xi_{m}\right) \\
=A i\left(\frac{x-x_{m}(z)}{x_{0}}+i \frac{a_{x} z}{k x_{0}^{2}}\right) \exp \left(\frac{a_{x}\left(x-x_{m}(z)\right)}{x_{0}}-i \frac{z^{3}}{12 k^{3} x_{0}^{6}}+i \frac{a_{x}^{2} z}{2 k x_{0}^{2}}+i \frac{x z}{2 k x_{0}^{3}}\right) \\
A i\left(\frac{y-y_{m}(z)}{y_{0}}+i \frac{a_{y} z}{k y_{0}^{2}}\right) \exp \left(\frac{a_{y}\left(y-y_{m}(z)\right)}{y_{0}}-i \frac{z^{3}}{12 k^{3} y_{0}^{6}}+i \frac{a_{y}^{2} z}{2 k y_{0}^{2}}+i \frac{y z}{2 k y_{0}^{3}}\right),
\end{gathered}
$$

the $A i\left(s_{m}\right)$ denotes the Airy function and the dimensionless transverse coordinates are $s_{x}=x / x_{0}$ and $s_{y}=y / y_{0}$, the normalized propagation distance are $\xi_{x}=z / k x_{0}^{2}$ and $\xi_{y}=z / k y_{0}^{2} . x_{0}=y_{0}=1.5 \mathrm{~mm}$ are normalization constants and $\mathrm{a}_{m=x, y}$ represents the decay factor acts as an exponential aperture function. Along the propagation direction z path, the ballistic trajectory [32-33] of the main lobe of Airy beam can be directly determined by:

$$
\left\{\begin{array}{l}
x_{m}(z)=\frac{z^{2}}{4 k^{2} x_{0}^{3}}+\theta_{x} z \\
y_{m}(z)=\frac{z^{2}}{4 k^{2} y_{0}^{3}}+\theta_{y} z
\end{array},\right.
$$

here, the $\theta_{m=x, y}$ denote the initial launch angles associated with the beam coordinate axis $\mathrm{x}$ and $\mathrm{y}$. It illustrates that the deflection of peak intensity from straight line is wavelength dependent [34].

The self-healing properties of Airy beam partially blocked by a finite opaque obstacle can be explained by Babinet's principle. It has been demonstrated that the robustness of the self-healing of non-diffracting optical beams in adverse environments, such as scattering media and turbulence, are corresponding to the internal power flow described by Poynting vector and angular momentum [30]. The theoretical calculating evolutions of self-bending [35] propagation configuration and self-reconstruction as a function of propagation distance are shown in figure 1(a) and 1(b). Here, the two wings of the initial Airy beam are truncated by different decay factors $a_{x}=0.01$ and $a_{y}=0.2$. Figure 1 shows that oscillating self-reconstruction dynamics of the partially truncated tailing of $y$-wing can be observed along the propagation path. Another $\mathrm{x}$-wing gradually decays into circumambience of free space.

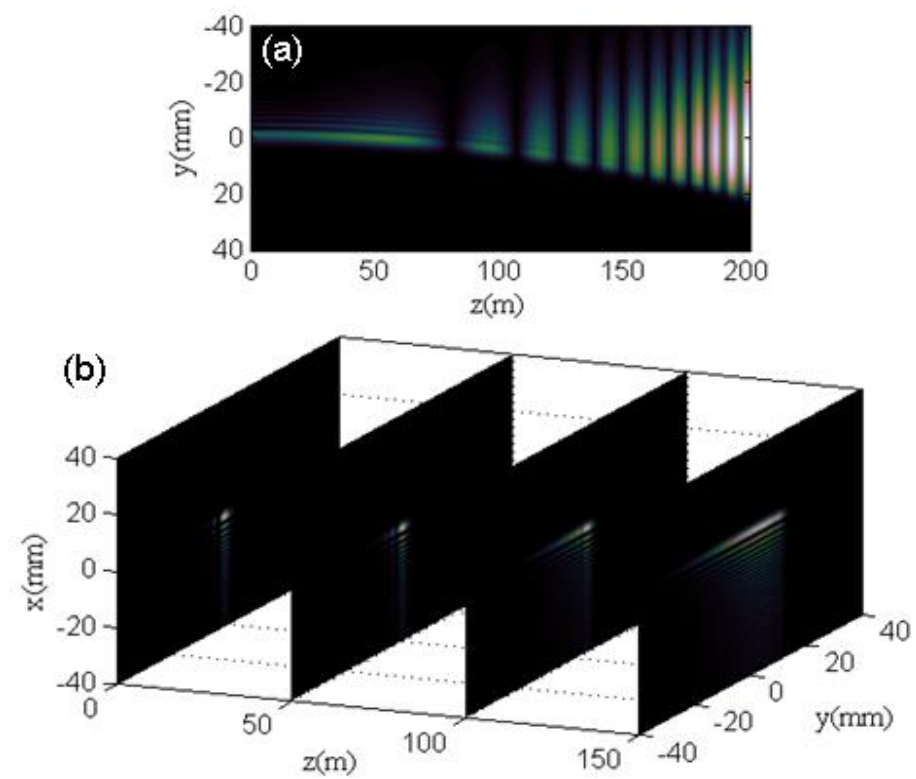

Fig. 1. (Color online) (a) The theoretical calculating self-bending propagation configuration of truncated Airy beam. (b) The self-reconstruction processes of truncated Airy beam. 


\section{The numerical results and discussions}

\subsection{The self-bending, ballistic trajectory and transversal accelerating}

The numerical simulation of self-bending of Airy beam is shown in Fig. 2(a). The yellow dash-line represents the ballistic trajectory of the main lobe according to the theoretical formula (4). The numerical results consistent with theoretical calculations well. Along the propagation path, the two wings of Airy beam developed into different propagating scenarios. In the transverse $\mathrm{x}$-direction, the intensity profile indicates that the beam has undergone an important transformation from Airy function to a Gaussian profile as can be seen in Fig. 2(b). In the transverse $\mathrm{y}$-direction, the configuration is absolutely different from that of transverse $\mathrm{x}$-direction. A self-similar Airy-shaped intensity profiles, featured by slowly decaying, oscillating tails, can be observed at different positions along propagation path shown in Fig. 2(c). As the continuing propagation, the intensity profile will finally become into a Gaussian shape as similar as that of $\mathrm{x}$-wing. The transversal accelerating of the Airy beam in the transversal plane including $\mathrm{x}$ and $\mathrm{y}$ directions independent on the truncation factors indicated in formula (4). It has been demonstrated that the accelerating trajectory can be controlled with optically induced refractive-index gradient to achieve the goal of propagation along arbitrary trajectory [36-37].
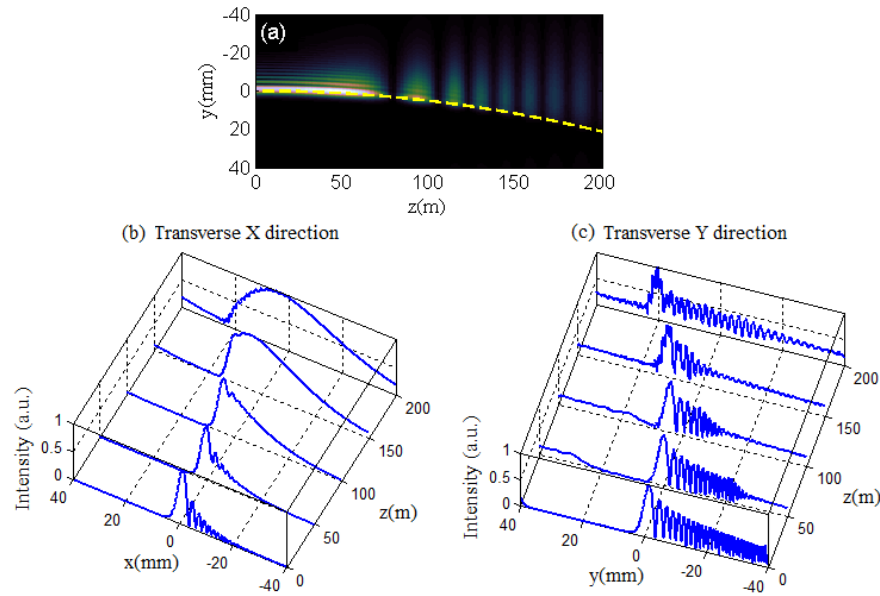

Fig. 2. (Color online) (a) The numerically calculated self-bending propagation configuration of truncated Airy beam, the yellow dash-line represents the ballistic trajectory of the main lobe according to the theoretical formula (4). The intensity profiles as a function of distance of truncated Airy beam, (b) corresponding to $\mathrm{x}$-direction and (c) corresponding to $\mathrm{y}$-direction.

\subsection{The self-healing of airy beams}

The self-healing property of Airy beam implies that the propagation is independent of the initial condition and is hardly sensitive to small phase fluctuation of the incident beam, resulting in the system develops towards a stable mode, at least if one observes its intensity profile at a finite-distance. The numerically calculated self-healing processes are shown in Fig. 3, which is in excellent agreement with Fig. 1(b) obtained from theoretical calculation. While this self-healing processes are different from that perturbed or blocked by a particle of the main lobe of Airy beam. The blocked Airy beam reconstructed from an interference field of its own two-wing side lobes. From the self-healing dynamics, it can be seen that y-wing propagates much longer distance compared with that of $\mathrm{x}$-wing, due to the former undergoes a special course of self-healing firstly before the diffraction propagation manner that $\mathrm{x}$-wing directly encountered.

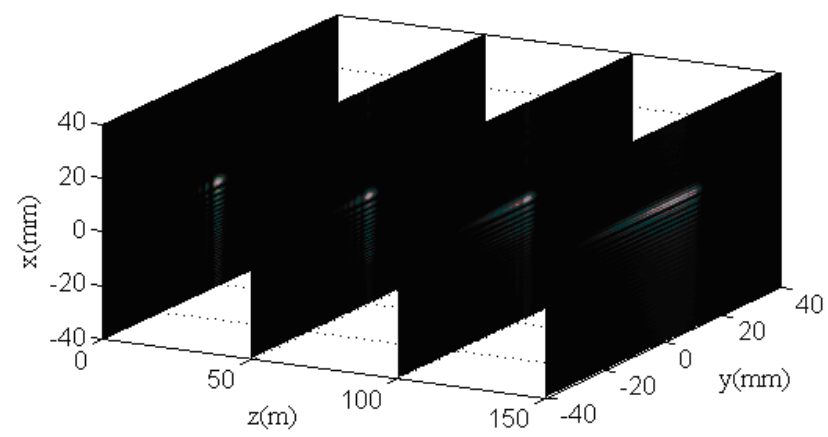

Fig. 3. (Color online) (a) The numerically calculated self-healing propagation configuration of truncated Airy beam with different decay factors $a_{x}=0.01$ and $a_{y}=0.2$. 
While when the decay factor is adjusted to $a_{y}=1$ and keep the $a_{x}$ not changed, an absolutely different accelerating propagation manner can be observed as shown in Fig. 4(a). The transversal acceleration and position shift of the main lobe occur only along the $x$-wing as can be seen in Fig. 4(b), the trajectory of the main beam becomes a straight line in $x$-direction but not curved along the cross angle $45^{\circ}$ in the $x-y$ plane enabling the self-bending trajectory of Airy beam. The y-wing exhibits a normal diffraction propagation similar as Gaussian beam in spite of the initial intensity profile with a slight oscillating tailing seen in Fig. 4(c).
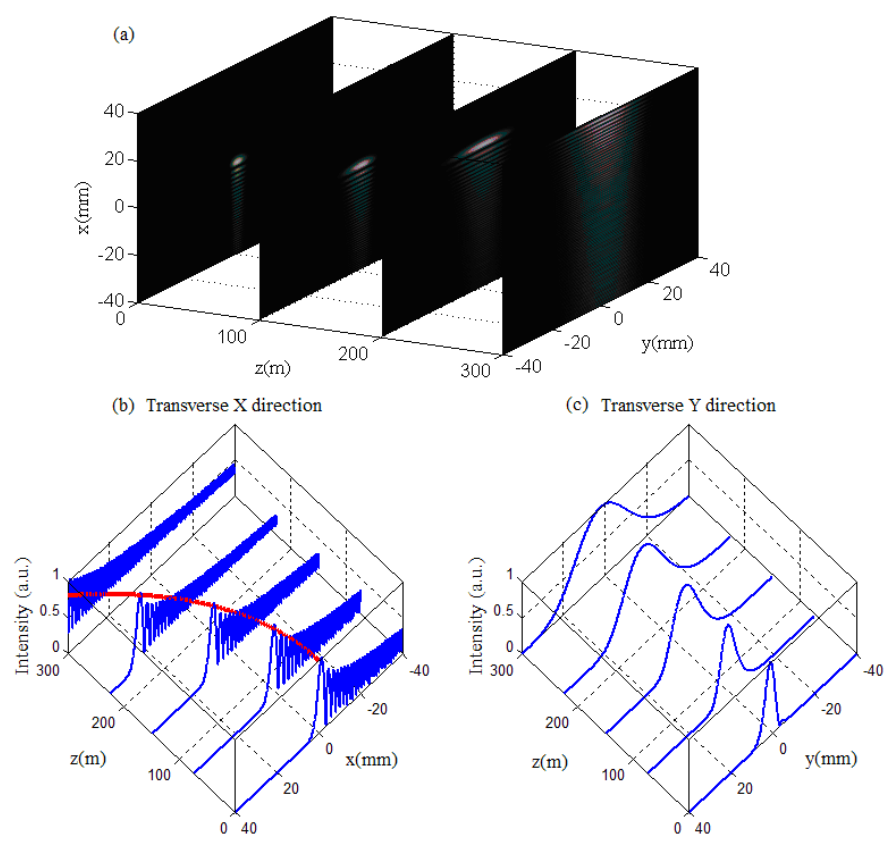

Fig. 4. (Color online) (a) The numerically calculated propagation configuration of truncated Airy beam with different decay factors $a_{x}=0.01$ and $a_{y}=1.0$. The intensity profiles as a function of distance, (b)

corresponding to $\mathrm{x}$-direction and (c) corresponding to y-direction. The red dash-line represents the ballistic trajectory of the main lobe in the $\mathrm{x}-\mathrm{z}$ plane.

\subsection{The pointing vector and energy flow}

We gain an insight into the evolution to explain the above observed patterns emanating from numerical calculation. When $a_{y}=1.0$, it means the y-wing of the beam is absolutely truncated by a Gaussian function window, and only the $\mathrm{x}$-wing of the beam evolution with a long oscillating tailing similar with Bessel beam. It is demonstrated that the spatial truncation function plays an important inherent influence on the extrinsic evolution of the ideal Airy beam. Furthermore, we conduct the practical analysis by using the Poynting vector $\vec{S}$ and energy flow to illuminate the field configuration. In the paraxial regime, $\vec{S}$ is given by:

$$
\vec{S}=\vec{S}_{z}+\vec{S}_{\perp}=\frac{1}{2 \eta_{0}}|u|^{2} \hat{z}+\frac{i}{4 \eta_{0} k}\left[u \nabla_{\perp} u^{*}-u^{*} \nabla_{\perp} u\right] \text {, }
$$

where the $\eta_{0}=\sqrt{\mu_{0} / \varepsilon_{0}}$ represents the impedance of free space. $\vec{S}_{z}$ and $\vec{S}_{\perp}$ denote the longitudinal and transverse components of the Poynting vector, respectively. In figure 5, we plot the Poynting vector, which intuitively demonstrates the internal energy flow during the transversal acceleration and self-healing processes, of the field at two different distances for $a_{x}=0.01$ and $a_{y}=0.2$. At the initial propagation stage $\mathrm{z}=50 \mathrm{~m}$, only small energy of $\mathrm{x}$-wing flows towards the lateral regimes along y direction, resulting in rebirth of the secondary side lobes from the y-wing and reformation of the newly formed coherent field in the internal cross regime. The external energy flows evidently facilitate the lateral acceleration and enable the self-bending of Airy beam along cross angle $45^{\circ}$ in the $x-y$ plane. As propagation continued, the reconstruction dynamic will be enhanced, i.e., after $z=110 \mathrm{~m}$, the complete regeneration of $y$-wing can be observed.

While when the y-wing is completely truncated as shown in figure 4 , most energy flow outward along the $\mathrm{x}$-wing and there is not enough energy efficiently compensate to realize the reformation the $\mathrm{y}$-wing. Therefore, an acceleration only occurs in the $\mathrm{x}$ direction and a straight line propagation can be obtained. 

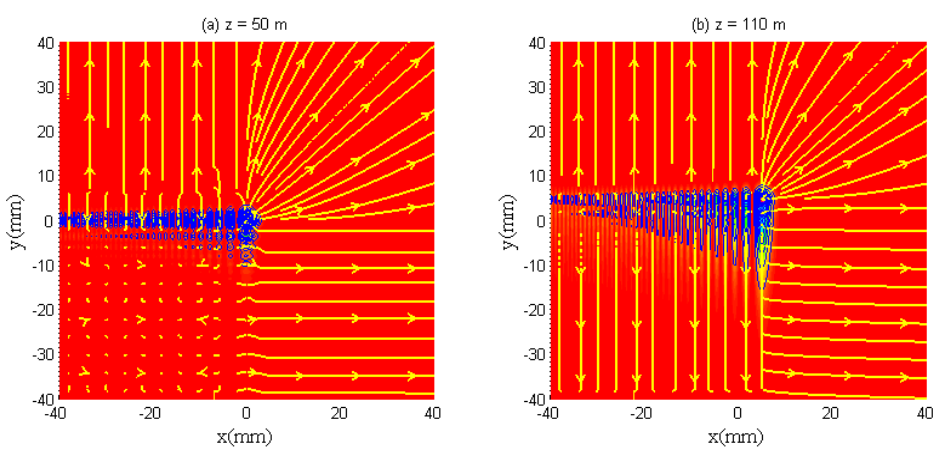

Fig. 5. (Color online) The calculated transverse energy flow at different distances for $a_{x}=0.01$ and $a_{y}=0.2$. (a)

$$
\mathrm{z}=50 \mathrm{~m} ; \text { (b) } \mathrm{z}=110 \mathrm{~m} \text {. }
$$

\section{Summary}

We have numerically demonstrated the self-healing peculiarity of Airy beam truncated by different decay factors. The self-healing dynamic processes of truncated Airy beams are different from that of blocked Airy beams. The wing with large truncation of Airy beam will propagate much longer distance than that of small truncated wing. While the Airy beam, one of the wings is complete truncated, can propagate along a straight line and neither self-bending nor self-reconstruction can be observed. It indicates that the truncation function has important influence on the propagation trajectory of Airy beam. The Poynting vector and energy flow are conducted to gain an insight into the physical mechanism.

\section{Acknowledgements}

The project was supported by Open Research Fund of State Key Laboratory of Pulsed Power Laser Technology, Electronic Engineering Institute (Grant Nos: SKL2013KF01 and SKL2015KF03); National Natural Science Foundation of China (Grant No: 61605223) and Dean fund of Hefei Institutes of Physical Science, Chinese Academy of Sciences (Grant No. YZJJ201506).

\section{References}

[1] Migule A. Bandres, Ido Kaminer, Matthew S. Mills, B. M. Rodríguez-Lara, Elad Geenfield, Morderchai Segev and Demetrios N. Christodoulides. "Accelerating optics beams", Optics \& Photonic News 2013, 30-37.

[2] Miguel A Bandres and B M Rodríguez-Lara, "Nondiffracting accelerating waves: Weber waves and parabolic momentum", New Journal of Physics 15, 2013, 013054.

[3] M. Clerici, D. Faccio, A. Lotti, E. Rubino, O. Jedrkiewicz, J. Biegert, P. Di Trapani, "Finite-energy, accelerating Bessel pulses", Optics Express 2008, 16(24), 19807-19811.

[4] Gil Porat, Ido Dolev, Omri Barlev, and Ady Arie, “Airy beam laser”, Optics Letters 2011; 36: 4119-4121.

[5] Pavel Polynkin, Miroslav Kolesik, Jerome V. Moloney, Georgios A. Siviloglou, Demetrios N. Christodoulides, "Curved Plasma Channel Generation Using Ultraintense Airy Beams" Science 2009, 324, 229-232

[6] Baumgartl J, Mazilu M, Dholakia K. Optically mediated particle clearing using Airy wavepackets. Nature Photonics 2008, 2 , 675-678.

[7] Peeter Piksarv, Andreas Valdmann, Heli Valtna-Lukner and Peeter Saari, “Ultrabroadband Airy light bullets”, Journal of Physics: Conference Series 2014, 497, 012003.

[8] Jérôme Kasparian and Jean-Pierre Wolf, "Laser Beams Take a Curve”, Science 2009, 324, 194-195.

[9] Noa Voloch-Bloch, Yossi Lereah, Yigal Lilach, Avraham Gover and Ady Arie, "Generation of electron Airy beams", Nature, 2013, 494,331-335.

[10] Ido Kaminer, Rivka Bekenstein, Jonathan Nemirovsky, and Mordechai Segev, "Nondiffracting Accelerating Wave Packets of Maxwell's Equations", Physical Review Letters 2012, 108(16), 163901.

[11] M. V. Berry and N. L. Balazs, "Non-spreading wave packet,” American Journal of Physics 1979, 47(3), 264-267.

[12] K.B. Oldham et al., "An Atlas of Functions, Second Edition”, Springer Science Business Media, LLC, 2009, Chapter 56,585-592

[13] S. Chávez-Cerda, "A new approach to Bessel beams," Journal of Modern Optics 1999, 46, 923-930.

[14] Planchon T A, Liang Gao, Milkie D E, Davidson M W, Galbraith J A, Galbraith C G, et al. "Rapid three-dimensional isotropic imaging of living cells using Bessel beam plane illumination". Nature Methods 2011; 8(5): 417-423.

[15] Siviloglou G A, Brokly J, Dogariu A, Christodoulides D N. "Observation of accelerating Airy beams". Physical Review Letters 2007; 99: 213901.

[16] Siviloglou G A, Christodoulides D N. "Accelerating finite energy Airy beams”. Optics Letters 2007; 32: 979-981.

[17] T. Ellenbogen, N. Voloch-Bloch, A. Ganany-Padowicz, and A. Arie, "Nonlinear generation and manipulation of Airy beam," Nature Photonics 2009, 3, 395-398.

[18] Berna Yalizay, Burak Soylu, and Selcuk Akturk, "Optical element for generation of accelerating Airy beams", Journal of the Optical Society of America A 2010, 27(10), 2344-2346.

[19] Don M. Cottrell, Jeffrey A. Davis, and Thomas M. Hazard, "Direct generation of accelerating Airy beams using a $3 / 2$ phase-only pattern", Optics Letters 2009; 34: 2634-2636.

[20] Miguel A. Bandres, "Accelerating parabolic beams", Optics Letters 2008, 33: 1678-1680. Torre, "Airy beams beyond the paraxial approximation", Optics Communications 2010, 283, 4146-4165.

[21] Luis Carretero, Pablo Acebal, Salvador Blaya, Celia Garcia, Antonio Fimia, Roque Madrigal, Angel Murciano, "Nonparaxial diffraction analysis of Airy and SAiry beams”, Optics Express, 2009, 17(25), 22432-22441. 
[22] Fred E. Bradley, "Development of an Airy stress function of general applicability in one, two, or three dimensions", Journal of applied physics, 1990, 67, 225-226.

[23] Paris Panagiotopoulos, Dimitris Papazoglou, Stelios Tzortzakis, and Arnaud Couairon, "Controlling high power autofocusing waves

[24] with periodic lattices", To be published in Optics Letters, July 2014, Doc. ID: 214241.

[25] Wei Wen, KehuiSong, YimingDong, MinYao, "Finite energy Airy-Hermite-Gaussian beam and its paraxial propagation", Optics \& Laser Technology 2013, 48, 28-34.

[26] P. Saari, "Airy Pulse-A New Member of Family of Localized Waves”, Laser Physics, 2009, 19(4), $725-729$.

[27] Chunyi Chen, HuaminYang, Mohsen Kavehrad, Zhou Zhou, "Propagation of radial Airy array beams through atmospheric turbulence", Optics and Lasers in Engineering 2014, 52, 106-114

[28] Ru-mao Tao, Lei Si, Yan-xing Ma, Pu Zhou, Ze-jin Liu, “Average spreading of finite energy Airy beams in non-Kolmogorov turbulence", Optics and Lasers in Engineering 2013, 51,488-492.

[29] Daryoush Abdollahpour, Sergiy Suntsov, Dimitrios G. Papazoglou, and Stelios Tzortzakis, "Spatiotemporal Airy Light Bullets in the Linear and Nonlinear Regimes", Physical Review Letters 2010; 105(25): 253901.

[30] John Broky, Georgios A. Siviloglou, Aristide Dogariu, and Demetrios N. Christodoulides, "Self-healing properties of optical Airy beams", Optics Express 16(17),12880-12891.

[31] Xiuxiang Chu, Guoquan Zhou, and Ruipin Chen, "Analytical study of the self-healing property of Airy beams", Physics Review A 2012, 85(1), 013815.

[32] G. A. Siviloglou, J. Broky, A. Dogariu, D. N. Christodoulides, "Ballistics dynamics of Airy beams", Optics Letters 2008, 33, 207-209.

[33] Yi Hu, Peng Zhang, Cibo Lou, Simon Huang, Jingjun Xu, and Zhigang Chen. "Optimal control of the ballistic motion of Airy beams", Optics Letters, 2010, 13, 2260-2262.

[34] J. E. Morris, M. Mazilu, J. Baumgartl, T. Čižmár and K. Dholakia, "Propagation characteristics of Airy beams: dependence upon spatial coherence and wavelength", Optics Express, 2009, 17(15), 13236-13245.

[35] Zhigang Chen, "Light Bends Itself into an Arc", Physics 2012, 5, 44

[36] Elad Greenfield, Mordechai Segev, Wiktor Walasik, and Oren Raz, "Accelerating Light Beams along Arbitrary Convex Trajectories", Physical Review Letters 2011; 106(21): 213902.

[37] Nikolaos K. Efremidis, “Airy trajectory engineering in dynamic linear index potentials”, Optics Letters, 2011, 36, 3006-3008. 